\title{
BONE TURNOVER MARKERS IN OLD OLD VS POSTMENOPAUSAL WOMEN
}

Preeti Nair ${ }^{\mathrm{A}}$, Kien Chan ${ }^{\mathrm{A}}$, Wee Yang Pang ${ }^{\mathrm{A}}$, Anupam Chauhan ${ }^{\mathrm{A}}$, EE Mun Lim ${ }^{\mathrm{B}, \mathrm{C}}$, Charles Inderjeeth ${ }^{\mathrm{A}, \mathrm{B}}$ Area Rehabilitation and Aged Care, North Metropolitan Health Service, Hospital Avenue,
Nedlands, Perth, WA 6009, Australia

${ }^{B}$ University of Western Australia, Perth, WA 6009, Australia PathWest Laboratory Perth WA

Postmenopausal women form the population base of most studies on osteoporosis interventions and outcomes. However the mechanism of bone loss appears to differ in senile osteoporosis. We performed a retrospective review of fasting metabolic bone studies (FBMS) done by $\mathrm{Cl}$ in the period from 2002 to 2012 , looking for differences in bone turnover markers between postmenopausal (age 50-65) and old old (age 75 and above) women. The results showed a significantly lower P1NP level in the old old group but no statistically significant difference in ALP or urine NTx/Cr ratio between the groups. This suggests that the bone turnover profile of the two groups are different, with lower formation in the old old compared to postmenopausal group, and similar resorption between the two groups. This may have implications for future research into osteoporosis treatment in the old old.

\section{Background}

It is increasingly recognised that osteoporosis is not a homogenous disease. Riggs et al identified two distinct types of osteoporosis in 1983, with different pathophysiology, patterns of bone loss and fracture types. ${ }^{1}$

Post-menopausal (PM) osteoporosis is triggered by withdrawal of the effect of oestrogen on bone, which leads to a sharp acceleration of bone turnover with an imbalance towards excessive osteoclastic activity, resulting in rapid net bone loss that is disproportionately higher in trabecular bone and increases the risk of vertebral fracture predominantly

Senile osteoporosis, on the other hand, is a phenomenon described in the old old (usually after the age of 75) and affects both genders. It is characterised by a shift from osteoblastogenesis towards adipogenesis in the bone marrow ${ }^{2}$, with consequent paucity of bone formation vis-à-vis resorption, affecting both cortical and trabecular bone and leading to increased hip fractures in the elderly. As the world population ages, senile osteoporosis, with its attendant fracture patterns and therapeutic implications, is expected to play an increasingly central role.

The use of bone turnover markers in osteoporosis has been extensively investigated including its role in diagnosis, prognostication of the risk of future bone loss and fracture risk, selection of initial therapy, monitoring of efficacy and monitoring of compliance. It is well established that bone turnover makers, especially resorption, are disproportionately increased in postmenopausal osteoporosis, resulting in net bone loss. ${ }^{3}$ However, data on senile osteoporosis is limited and there is virtually no comparison data available.

\section{Aim and Hypothesis}

We aim to compare bone turnover markers in a postmenopausal group to the old old We hypothesise that the difference in their profiles would reflect the differences in underlying mechanisms of osteoporosis - increased resorption in the postmenopausa group versus decreased formation and possibly resorption (stasis) in the old old.

\section{Methodology}

Retrospective audit of all fasting metabolic bone studies (FMBS) performed by the author (CI) in the outpatient clinic during the period 2002-2012. Patients' case notes and clinic letters were reviewed for exclusion criteria and the included cases were divided into the postmenopausal (age 50-65)(PM) and old-old (age 75 and above)(OO) groups. Where multiple FMBS were performed on the same patient, we included only the earliest record.

\section{Exclusion criteria}

- Male gender

- Prior use of osteoporosis medication (excluding Calcium and Vitamin D supplements)

- Prior or current use of long-term steroids (>3 months)

- Any fracture within the preceding 6 months

- Any disorders that may affect bone turnover: Paget's disease, Ankylosing Spondylitis, radiation induced osteoporosis, long term paraplegia, bone malignancy/metastases, primary hyperparathyroidism

- Insufficient information to verify criteria

Statistical analysis was performed using the IBM SPSS Statistics 20 package. Bone resorption marker urinary NTx/Cr ratio, and bone formation markers serum ALP and P1NP levels (where available) were compared across the two groups using the independent sample t-test. Univariate and multivariate analyses of the variables impacting these parameters were also analysed.
Results

976 FMBS were performed by $\mathrm{Cl}$ between January 2002 and March 2012. 55 patients met the predefined criteria and were included in the final analysis.

\begin{tabular}{lccc} 
& $\mathrm{PM}(\mathrm{n}=\mathbf{2 8})$ & $\mathrm{OO}(\mathrm{n}=\mathbf{2 7})$ & Significance \\
\hline Age & $58.7 \pm 4.3$ & $79.7 \pm 3.5$ & $\mathrm{p}<0.001$ \\
Albumin & $44.5 \pm 2.7$ & $42.6 \pm 2.27$ & $\mathrm{P}=0.009$ \\
Vitamin D & $65.8 \pm 24.3$ & $60.2 \pm 32.1$ & $\mathrm{p}=0.47$ \\
PTH & $6.8 \pm 2.5$ & $8.2 \pm 5.4$ & $\mathrm{p}=0.21$ \\
Serum Calcium (adjusted) & $2.29 \pm 0.07$ & $2.32 \pm 0.09$ & $\mathrm{p}=0.13$ \\
Serum Creatinine & $70.3 \pm 11.0$ & $72.0 \pm 14.2$ & $\mathrm{p}=0.60$ \\
Urinary Creatinine & $6.0 \pm 4.0$ & $5.7 \pm 2.9$ & $\mathrm{p}=0.72$ \\
Urinary Calcium/Creatinine ratio & $0.3 \pm 0.2$ & $0.5 \pm 0.4$ & $\mathrm{p}=0.07$ \\
Serum P1NP & $73.9 \pm 43.3$ & $41.6 \pm 12.1$ & $\mathrm{p}=0.037$ \\
Serum ALP & $88.7 \pm 29.0$ & $78.3 \pm 18.0$ & $\mathrm{p}=0.127$ \\
Urinary NTx/Creatinine ratio & $40.0 \pm 18.6$ & $42.8 \pm 15.5$ & $\mathrm{p}=0.554$ \\
\hline
\end{tabular}

* PTH - Parathyroid hormone, P1NP - N-terminal propeptides of procollagen type $1, A L P$ - Alkaline phosphatase, NTx - N-telopeptides of type 1 collagen

Neither univariate nor multivariate associations for possible confounders (Vitamin $D$ PTH, plasma Creatinine and Calcium) were significantly associated with $\mathrm{NTx} / \mathrm{Cr}(\mathrm{p}>0.05)$

\section{Discussion}

Bone formation marker P1NP was significantly lower in the old old group. This supports the hypothesis by Riggs et al $^{1}$ regarding reduced bone formation in senile osteoporosis. This may have implications for treatment in the old old with predominant cortical osteoporosis. Anabolic treatments may be preferable to anti-resorptive therapies. ${ }^{4}$ More research is required in this therapeutic area.

The lack of any significant difference in serum ALP may be due to the lab test measuring total ALP rather than bone-specific ALP, which can also be affected by other systemic conditions including hepatic disease.

We found no significant difference in bone resorption markers between the two groups. This was unexpected, given our hypothesis that the predominant pathophysiology in PM osteoporosis is one of predominant resorption (osteoclastic activity) compared to senile osteoporosis, where both formation and resorption were expected to be reduced (stasis) Possible reasons include an increase in bone remodelling sites in the old old following prolonged resorption, rather than an increased rate of resorption at each individual site. ${ }^{5}$ Furthermore, urine NTx/Creatinine ratios are influenced by confounding factors that affect serum Creatinine, which is influenced in opposite directions by age-associated deterioration in renal function (increases $\mathrm{Cr}$ ) and muscle bulk (decreases $\mathrm{Cr}$ ).

\section{References}

1. Riggs BL, Melton LJ III. Evidence for two distinct syndromes of involutional osteoporosis. Am J Med 1983;75:899-901

2. Duque G, Troen BR. Understanding the mechanisms of senile osteoporosis: new facts for a major geriatric syndrome. J Am Geriatr Soc 2008;56:935-941

3. Garnero P, Sornay-Rendu E, Chapuy MC, Delmas PD. Increased bone turnover in late postmenopausal women is a major determinant of osteoporosis. J Bone Miner Res 1996;11:337-349

4. Inderjeeth CA, Foo ACH, Lai MMY, Glendenning P. Efficacy and safety of pharmacological agents in managing osteoporosis in the old old: Review of the evidence. Bone 2009;44:744-751

5. Seeman E, Dalmas PD. Bone quality - the material and structural basis of bone strength and fragility. N Engl J Med 2006;354:2250-2261 